\title{
Diseño e implementación de sistemas de calidad con base en las Normas ISO 9000:2000
}

Martha Lucía Sarria Meléndez ${ }^{1}$

1 Maestría en Administración y Dirección de Empresas (MADE) Facultad de Ciencias Económicas y Empresariales de la UCA. Apdo. 69, Managua, Nicaragua.

\section{Recibido: abril 2006 / Aceptado: mayo 2006}

LAS EMPRESAS BUSCAN LA CERTIFICACIÒN ISO 9000:2000 PORQUE LOS clientes lo exigen, pues garantiza que brindan productos y/ o servicios de calidad consistente. Los beneficios que reciben las empresas son ventajas competitivas en el mercado, mayor uniformidad en las operaciones internas evitando desperdicios y redundancias; mejor calidad en los productos y servicios; menos auditorias por cliente; más énfasis en el mejoramiento continuo; reducción de costos y mejoras en el rendimiento de las instalaciones y mejor documentación. Por ello, las empresas han iniciado procesos para implementar la norma ISO. Por su parte, las cámaras y el gobierno han iniciado programas para motivar su implementación, pues la eliminación de las barreras técnicas al comercio, el intercambio comercial fluido, y la implementación de tratados de libre comercio señalan que las barreras arancelarias desaparecerán, y se transformarán en barreras basadas en normativas. Este trabajo ofrece los pasos necesarios para alcanzar la norma.

Palabras clave: control de calidad, normalización, ventaja competitiva (comercio)

\section{Introducción}

La necesidad de estandarizar internacionalmente la calidad en la producción de bienes y servicios hizo necesario crear en 1946 el Organismo Internacional de Estandarización (ISO por sus siglas en ingles), que en la actualidad cuenta con 133 estados miembro y es representado a través de organismos nacionales de normalización. En Nicaragua, el SICAN (Sistema Integrado de Calidad de Nicaragua).

En un mundo competitivo, de libre comercio y acelerado desarrollo tecnológico, los países en vías de desarrollo necesitan estandarizar la calidad de los procesos productivos de bienes y servicios. La implementación de ISO es esencial para competir en el mercado internacional. 
La norma ISO 9000:2000 define la calidad como "el conjunto de las características de un producto o servicio que tienen que ver con su capacidad de satisfacer las necesidades definidas o implícitas"i. Estas normas pueden aplicarse prácticamente en cualquier compañía, desde fabricantes de productos hasta proveedores de servicios. No están diseñadas específicamente para un producto o industria determinada, por lo que se adaptan a cualquier necesidad.

Esta investigación es descriptiva y trata sobre la implementación de las normas ISO 9000:2000, mediante el establecimiento de sistemas de aseguramiento de calidad documentados. Se pretende dotar a las empresas de una metodología que sirva como herramienta para iniciar el proceso de certificación ISO 9000:2000, incluyendo la descripción de los requerimientos de la norma y el resumen de los beneficios tangibles e intangibles que se logran.

\section{Requisitos para implementar las Normas ISO 9000:2000}

Para que a una entidad le sea otorgada la certificación ISO 9000:2000, debe cumplir con la siguiente lista de requisitos:

Responsabilidad de la dirección: Para precisar la política de calidad y definir los recursos; personal con responsabilidades definidas. Los directivos deben participar activamente en el proceso de certificación.

Sistema de calidad: Para asegurar que los productos estén conformes con las especificaciones de la empresa y con las expectativas del cliente. Se requiere un manual de calidad, procedimientos y un plan de calidad.

Revisión de contratos: Para conocer las necesidades y exigencias del cliente, cumplir con sus expectativas y, si es posible, superarlas. Hay que documentar los requerimientos del cliente.

Control de documentos y datos: Hay que asegurar la validez de los documentos que circulan por la entidad: procedimientos e instrucciones de calidad, o especificaciones de productos terminados o materias primas.

Control de proveedores: Para que los productos y servicios adquiridos se adapten a las especificaciones internas, hay que evaluar a proveedores, al personal de compras y revisar la documentación (especificaciones) existente.

Control de productos: Control detallado del producto suministrado por los clientes (esto aplica para las empresas de servicios).

Trazabilidad: Para reconstruir la historia de un bien ó servicio que haya pasado por la entidad, por medio de una identificación registrada. La entidad debe contar con mecanismos de identificación de productos en la recepción, en las etapas del proceso productivo, almacenamiento ó entrega, instalación ó realización, cuando se trata de servicios. A la vez, deben definirse los medios de identificación que indiquen la conformidad o inconformidad.

Control de procesos: Los procesos deben controlarse de manera que la generación del producto ó servicio no conforme se reduzca al mínimo posible.

Control de instrumentos de medición, inspección o prueba: Deben elaborarse procedimientos y planes de calibración para promover confianza en los sistemas para alcanzar las especificaciones.

Control de no conformidades: El personal autorizado llevará a cabo la disposición del producto no conforme. Debe determinarse si el producto no conforme es apto para reproceso 
o no, que utilidad se le dará si puede dársele otro propósito, etc.

Acciones correctivas y preventivas: Identificar y eliminar las causas de la no conformidad. Las acciones preventivas tienen la misma finalidad de las acciones correctivas, con la diferencia de que pueden planificarse.

Manejo, almacenamiento, embalaje preservación y entrega.

Control de registros: Garantiza la preservación de la información generada en los distintos procesos por un tiempo definido.

Auditorias internas de calidad: Para verificar el cumplimiento de las actividades de calidad con respecto al plan de calidad definido para la entidad.

Capacitación: Para tener personal competente y calificado para las diferentes actividades del sistema.

Servicio post venta.

Técnicas estadística: Para analizar las tendencias y tomar decisiones.

En pocas palabras, el sistema de aseguramiento de calidad basado en las normas ISO se resume en los siguientes principios:

1. Escribir lo que se hace: Diseño del sistema documental.

2. Hacer lo que esta escrito: Cumplir con los procedimientos establecidos en el sistema documental.

3. Registro de lo que se hizo: Guardando informes por un tiempo prudencial.

4. Verificar: Auditorias continuas.

5. Mejora continua: Actuar sobre la diferencia.

\section{Revisión de documentación y diagnóstico}

El proceso de certificación inicia con la evaluación de los documentos existentes, para conocer la situación de la documentación en la entidad y relacionarla con las necesidades de la norma ISO 9000:2000. Para ello, debe elaborarse una guía de diagnóstico del sistema de calidad actual de la entidad. Uno de los objetivos del informe resultante es presentar una guía de autodiagnóstico (GAP análisis ó análisis de brecha) que permitirá a entidades pequeñas, medianas ó grandes valorar la documentación de sus sistemas de aseguramiento de calidad. El informe deberá contener los documentos evaluados y la información obtenida aplicando la guía de autodiagnóstico (existencia; conformidad de los documentos con respecto a los requerimientos; utilidad de la misma; cuáles deben redactarse, etc.). Esta etapa sirve para determinar los documentos que debe tener una empresa para garantizar que los procesos se lleven a cabo bajo condiciones controladas de operación.

Las normas ISO 9000:2000 ofrecen la posibilidad de adaptarse a las características de cada entidad en cuanto a tamaño, actividad, complejidad e interacción entre los procesos e instrucción del personal. Será necesario definir qué elementos de la norma ISO 9000:2000 son aplicables a la organización y estudiar las regulaciones específicas del sector para determinar que responden a esos requerimientos legales.

\section{Elaboración del sistema documental}

Su objetivo es establecer los elementos generales necesarios para la elaboración del sistema 
documental. En esta fase deben llevarse a cabo tres actividades:

Jerarquizar la documentación y clasificarla con un criterio único: Generalmente, el criterio de pirámide de la ISO 10013:2000: Primer nivel, manual de calidad; segundo nivel: procedimientos; tercer nivel: instrucciones y especificaciones; cuarto nivel: control de registros. Asimismo, el criterio que clasifica los documentos regulatorios en tres niveles: primero, manual de calidad; segundo, procedimientos generales, tercero, procedimientos específicos, y otros documentos.

Definición de autoridad y responsabilidades en la de elaboración de documentos por nivel: En esta actividad debe involucrarse a todo el personal, por lo que debe ser desplegada por toda la organización según niveles jerárquicos establecidos y la estructura organizativa existente.

Definición de la estructura y formato del manual de calidad: El manual de calidad describe la estructura de la compañía, su enfoque para satisfacer las necesidades de sus clientes, la estructura de la documentación y la política y responsabilidades de la gerencia para cumplir los requisitos de la norma.

\section{Elaboración de los documentos}

Esta etapa es esencial en el proceso de implementación del sistema de calidad que se fundamenta en el material documentado. Los documentos bien elaborados ayudan a detectar los errores reiterados que suelen ser obviados por falta de capacitación o por descuido del personal. Los documentos deben estar redactados en lenguaje asequible y claro para todo el personal. Quienes se encarguen de la redacción deberán tener un nivel jerárquico adecuado y estar relacionados con el proceso productivo. Las actividades son: elaboración de los procedimientos generales; elaboración del manual de calidad; elaboración otros documentos siguiendo un plan trazado de antemano; revisión y aprobación todos los documentos por parte del personal competente autorizado.

Para implantar el sistema de documentación hay que definir un cronograma que tenga en cuenta las características de la organización y sus recursos. La documentación aprobada debe ser distribuida a las distintas áreas inmediatamente. Asimismo, debe revisarse y mejorarse continuamente sistema de calidad mediante un plan de auditorias internas que detecten y corrijan las fallas para implementar acciones correctivas y preventivas.

\section{Esquemas orientados a protección ambiental}

En Octubre de 1996, la Organización Internacional de Normalización promulgó una serie de estándares internacionales para gestión ambientalllamados ISO 14000 que han revolucionado la forma en que la industria y los gobiernos manejan los asuntos ambientales.

ISO 14000 provee un lenguaje común para la gestión ambiental mediante el establecimiento de un marco para la certificación de sistemas de gestión ambiental por tercera persona. ISO 14000 sigue los pasos de ISO 9000, una serie de estándares internacionales establecidos en 1987 para sistemas de gestión de calidad.

Si el éxito y la aceptación que han tenido los estándares ISO 9000 son algún indicador, en 
poco tiempo el conocimiento y la adopción de ISO 14000 llegará a ser una realidad para compañías que buscan el desarrollo de sus negocios y su mantenimiento en la vanguardia del mercado global.

El personal de Environment International Ltd. por ejemplo tiene un extenso conocimiento de los estándares ISO 14000 y ha ofrecido asesoramiento en una extensa variedad de temas relacionados con la gestión ambiental.

Apoyándose en la experiencia de su equipo en evaluaciones de riesgos ambientales, derecho ambiental, ecología, gestión de recursos naturales y política ambiental, Environment International Ltd. adopta un enfoque interdisciplinario para la gestión ambiental e ISO 14000 alejándose del carácter unidimensional de la mayoría de los consultores.

Haciendo uso de su diversidad profesional, los expertos de Environment International Ltd. trabajan desde las perspectivas científica, legal y de gestión en la identificación de riesgos ambientales en la empresa y en el desarrollo de sistemas de gestión ambiental efectivos para minimizar esos riesgos.

La diversidad de idiomas con que cuenta, así como su red de contactos domésticos e internacionales hacen de Environment International Ltd. un instrumento eficaz para la industria en la creación de estrategias para la gestión ambiental creativas, rentables y prácticas tanto en los Estados Unidos como en el extranjero.

Los científicos, abogados, especialistas en gestión ambiental y auditores certificados de ISO 14000 de Environment International Ltd. ofrecen un amplio espectro de servicios de consultoría de ISO 14000 entre los que se incluyen: auditorias ambientales; preparación de directores y empleados; desarrollo y evaluación de sistemas de gestión ambiental; desarrollo de protocolos legales y regulatorios; preparación para la adopción de ISO 14000

Hay muchas razones por las que una empresa debería considerar seriamente la implantación de sistemas de gestión ambiental, las auditorias de ISO 14000 y la certificación ISO 14000. Quizás la más poderosa es la demanda del mercado de productos "verdes", como se han demostrado en las controversias relacionadas con McDonalds y el uso de recipientes de poliestireno, la producción de atún y las capturas de delfines.

Según un informe reciente, realizado por una importante compañía consultora, cuando el precio y la calidad son iguales, el 76\% de los norteamericanos están dispuesto a cambiar a un producto asociado con una buena causa. El activismo ambiental empresarial, sugiere la consultora, actuará como rompedor del empate competitivo. Tendencias similares se dan en Europa. Un estudio realizado por una multinacional norteamericana descubrió que el tercer factor en las decisiones de compra, después de precio y calidad, es la percepción del consumidor en cuanto a la responsabilidad ambiental empresarial. Compañías certificadas bajo ISO 14000 pueden apuntar a esta certificación como prueba de una política ambiental positiva y, por tanto, ganar un puesto aventajado en el mercadeo de sus productos.

El acceso al mercado puede asimismoverse afectado por ISO 14000. Aunquela implementación de ISO 14000 es voluntaria, la certificación bajo estos estándares puede convertirse en un 
prerrequisito legal para participar en concursos y en el mercado en la Unión Europea y otras partes del mundo.

La certificación ISO 14000 puede satisfacer los requisitos de las regulaciones de Unión Europea en cuanto a sistemas de gestión ambiental. En Estados Unidos, agencias del gobierno tales como el Departamento de Energía están considerando seriamente implantar las Normas de ISO 14000 como requisito para sus contratistas.

Además de mejorar la competitividad, un sistema de gestión ambiental ISO 14000 puede ayudar a las empresas a economizar tiempo y dinero mejorando sus operaciones y facilitando las relaciones con las agencias del gobierno.

Con un sistema de gestión ambiental internacionalmente reconocido, las empresas pueden identificar más eficientemente problemas de seguridad y medio ambiente que se den en sus establecimientos, disminuir su potencial de responsabilidad y reducir el riesgo de accidentes graves.

Las auditorias ambientales pueden asimismo localizar prácticas ambientales ineficaces ó costosas en áreas tales como la producción industrial y el manejo de desperdicios. Finalmente, la certificación ISO 14000 puede disminuir las cargas legales de las empresas. Por ejemplo, según las guías del Departamento de Ecología del estado de Washington, los establecimientos requeridos de preparar planes de prevención de contaminación o actualizaciones del plan quinquenal pueden cumplir estos requerimientos demostrando que tienen un sistema de gestión ambiental ISO 14000.

La certificación bajo los estándares puede resultar en menor severidad por parte de las agencias reguladoras del gobierno tras un accidente medioambiental, ya que tal certificación puede servir como prueba de los esfuerzos positivos para ser una empresa responsable ambientalmente. Por tanto, aunque las demandas del mercado pueden ser el factor principal para considerar la implementación de ISO 14000, se puede añadir un número de beneficios que hacen que las auditorias y certificación bajo los estándares sean favorables (BULLTEK Ltd.).

\section{Esquemas relacionados a Responsabilidad Social (Seguridad e Higiene)}

SA 8000 (Social Accountability 8000) y OHSAS 18001 (Sistema de Gestión de la Prevención de Riesgos Laborales) son normas orientadas hacia la responsabilización social de las empresas.

En los países desarrollados, que concentran el mayor consumo de bienes del mundo, hay una preocupación creciente sobre los aspectos éticos de las empresas fabricantes de esos bienes. Sobre la base de la Declaración Universal de Derechos Humanos, las Convenciones de la Organización Internacional del Trabajo (OIT), la Convención de las Naciones Unidas sobre los Derechos de los Niños, y la Convención de las Naciones Unidas sobre la Eliminación de Todas las Formas de Discriminación contra la Mujer, se ha desarrollado una norma reconocida internacionalmente, la SA 8000. 
Esta norma tiene 9 áreas clave, sobre las que fija requisitos para las políticas, procedimientos y prácticas de las compañías en lo relativo a su responsabilidad social: No emplear a menores; No emplea mediante trabajo forzado; aseguramiento de las condiciones de salud y seguridad; libertad de asociación y derecho a convenios colectivos; ausencia de todo tipo de discriminación y acoso; ausencia de castigos corporales, abusos verbales o coerción; apropiada cantidad de horas de trabajo; retribución justa; implementación de un sistema de gestión de la responsabilidad social.

La forma de implementación del Sistema de Gestión de la Responsabilidad Social es similar al de la norma ISO 9001, y los elementos para asegurar su funcionamiento son comunes: documentación, registros, acciones correctivas y preventivas, mejoramiento continuo.

Una vez que se ha asegurado la implementación de sus requisitos, la empresa también puede optar por una certificación realizada por organismos acreditados. Esta certificación permite demostrar a los clientes y consumidores que la empresa tiene un Sistema de Gestión de la Responsabilidad Social implementado sobre la base de normas éticas reconocidas internacionalmente (SA 8000-Sistema de Gestión de la Responsabilidad Social).

OHSAS 18001 es una herramienta que ayuda a las empresas a identificar, priorizar y gestionar la salud y los riesgos laborales como parte de sus prácticas normales de negocio. La norma permite a la empresa concentrarse en los asuntos más importantes de su negocio. OHSAS 18001 requiere que las empresas se comprometan a eliminar o minimizar riesgos para los empleados y otras partes interesadas que pudieran estar expuestas a peligros asociados con sus actividades, y a mejorar de forma continuada como parte del ciclo de gestión normal.

La norma se basa en el conocido ciclo de sistemas de gestión de planificar-desarrollarcomprobar-actuar (PDCA) y utiliza un lenguaje y una terminología familiar propia de los sistemas de gestión.

OHSAS 18001 tiene como objetivos establecer, mantener y mejorar un sistema de gestión de la salud y seguridad laboral para asegurar la conformidad con la política y para demostrar tal conformidad a los demás. Es la principal conductora del sistema de gestión OHS y establece la estrategia "de comportamientos" de la compañía. Debe estar de acuerdo con la naturaleza y escala de los riesgos OHS de la compañía y ha de incluir el compromiso de mejora continua, la conformidad con la actual legislación OHS aplicable, y ha de ser comunicada a los empleados y puesta a disposición de las partes interesadas.

Varios elementos han de ser considerados para poner en marcha un Sistema de Gestión de Seguridad y Salud Laboral. Entre estos se incluyen: identificación de los peligros y evaluación de los riesgos; cumplimiento legal; consistencia en los compromisos; emergencia; gestión de suministradores y subcontratistas.

Los tres principios fundamentales, aunque son contra las que más se ha pecado, antes de comenzar el proceso son: leer la norma cuidadosamente y en detalle y darse cuenta de los "debe"; ver lo que se tiene y proponerse una mínima burocracia; leer la norma en una de las 
lenguas oficiales, ya que algunas traducciones no son precisas (Sistema de Gestión de la Prevención de Riesgos Laborales: OHSAS 18001).

\section{Implementación de la Norma ISO en Nicaragua}

ElBID, por medio de la CADIN, ha ofrecido financiamiento para un proyecto de modernización organizacional, cuyo objetivo es promover la implementación de sistemas de calidad documentados bajo las directrices de las normas ISO.

La empresa IMPASA se planteó el objetivo de obtener la certificación como parte de una estrategia para abrir nuevos mercados para sus productos, y para aumentar su competitividad. El proceso de implementación se llevó a cabo en el tiempo establecido de 13 meses, y representó un costo aproximado de 67,000.oo dólares de los que la empresa asumió el 35\%, y el 65\% restante fue financiado por el BID a través de la CADIN.

Las mayores dificultades surgieron por la resistencia del personal al cambio de estrategia, pero fueron solucionadas con capacitación, recomendada fuertemente por los asesores, y en algunos casos, reorganización del personal. El cambio no generó problemas laborales, ni importantes gastos por requerimiento tecnológico, pero sí hubo que fortalecer el área de ventas.

Con la certificación ISO 9000:2000 la empresa ha reducido costos de operación, reclamos y reproceso. La empresa ha obtenido un mejor posicionamiento en el mercado y aumentado su facturación.

La empresa también fue certificada por ICONTEC, fue seccionado por el proyecto BID/ CADIN. La certificación de esta empresa se de dio como parte de una estrategia del grupo corporativo, que tiene como meta certificar a todas sus empresas miembro.

Otra empresa que buscó la certificación del sistema de gestión de la calidad es Nicaragua Sugar Estates Limited conforme a la norma ISO 9001 versión 1994, que fue alcanzada en 14 meses, cumpliendo con el cronograma de implementación. Representó un costo aproximado de 75,000.0o dólares y la inversión realizada para obtener y mantener la certificación del sistema fue asumida completamente por recursos propios.

La principal dificultad surgida en el proceso de implementación fue la reacción al cambio de parte del personal, pero el problema se solucionó con sensibilización y capacitación del personal involucrado. La certificación incluyó todo el proceso de producción azucarera, desde la recepción de materia prima, hasta las ventas, pero no se incluyó el proceso de generación eléctrica.

Entre los beneficios obtenidos por la empresa se destaca un mayor control y consistencia de los procesos, que en su mayoría es proporcionado por la documentación generada, donde se encuentra la descripción general de los procesos, definición de parámetros y control de evidencias a través de registros. 
Se definieron las principales responsabilidades en los procesos, que están claramente plasmadas en los documentos del sistema de gestión de calidad, así como la identificación de los principales indicadores de gestión.

La empresa fue certificada por Lloyd's Register Quallity Assurance, seleccionado por su vasta experiencia y prestigio internacional, además de su reputación de ser los más exigentes.

Según la directiva de la empresa, la certificación sólo puede lograrse si la alta dirección está convencida de la estrategia y respalda todos los aspectos del proceso.

La directiva de Café Soluble S.A. consideró la obtención de la certificación ISO 9000:2000 como parte de una estrategia para ganar competitividad. El proceso se extendió por un período de dieciocho meses, dentro del tiempo definido para alcanzar el objetivo, y representó un costo total aproximado de 68,500.oo dólares, incluyendo costos de asesoría $(38,000.00$ dólares); costo de capacitación (9,500.oo dólares); y costo de la certificación (17,000.00 dólares), inversión que fue totalmente asumida por la empresa.

El problema de reacción al cambio ha estado presente en prácticamente todas las empresas, y Café Soluble S.A. no estuvo exenta, pero el problema también fue resuelto con capacitación y sensibilización del personal involucrado. En cuanto a los costos por requerimientos tecnológicos, no fueron significativos.

Entre los principales beneficios obtenidos por la empresa como consecuencia de la certificación son: un mejor control de procesos, incremento en la eficiencia y productividad operacional, mejora de la calidad del producto, y apertura de nuevos mercados. Se ha logrado una mejor conciencia del personal hacia la calidad, objetivos, misión y política de la empresa.

La empresa fue certificada por Bureau Veritas, escogido por la directiva por su calidad de servicio, y recomendaciones hechas por otras empresas.

La directiva recomienda a otras empresas a emprender el proceso de certificación de forma planificada, pero sobre todo realizando un trabajo en equipo que involucre a todo el personal.

El ingenio Monte Rosa fue adquirido en 1999 por Pantaleón Sugar Holding de Guatemala. En 2003, la nueva directiva consideró obtener la certificación ISO 9000 como parte de una estrategia para aumentar su competitividad. Al momento de redactar este trabajo, la empresa aún se encontraba en el proceso de certificación, que se definió para catorce meses, pero se cree se extenderá a dieciséis.

El costo total de la certificación se estima oscilará entre 40,000.00 y 60,000.oo dólares, pues aún no se elige al organismo certificador, lo que hace variar tanto el aproximado. Esta inversión está siendo asumida totalmente por la empresa.

La principal dificultad surgida en el proceso ha sido la resistencia al cambio, sobre todo por 
el exceso de trabajo que genera el proceso; por otro lado, la falta de capacidad técnica del personal no ha representado una limitante puesto que el personal ha recibido capacitación suficiente.

Es necesario que una persona que se dedique exclusivamente a liderar el proceso, aunque las demás labores puedan ser desarrolladas por el personal de la empresa, por lo que los requerimientos de recursos humanos significan un costo considerable.

Las inversiones de índole tecnológico no son indispensables, pero es recomendable algún tipo de software que apoye en el manejo de la documentación e indicadores; la empresa aun no lo adquiere pero está en evaluación actualmente.

Entre los beneficios obtenidos por la empresa se advierte ya una mayor conciencia del personal hacia la calidad, la identificación de proceso y la formulación de indicadores. Se ha logrado documentar aproximadamente el $60 \%$ del proceso, es un proceso que aun continúa.

La empresa recomienda, primeramente, buscar un organismo calificado para que aplique un diagnóstico a la empresa y que asesore en el diseño de un plan de implementación adecuado a las necesidades específicas de la entidad. Una empresa puede no estar certificada pero haber avanzado en mucho en aspectos requeridos para la certificación. Si no se cuenta con personal que domine la materia, será necesario buscar asesoría externa.

No se debe iniciar el proceso pensando solamente en la certificación, sino en el desarrollo de la funcionalidad de la empresa, trabajar a conciencia buscando que el sistema que se implemente sea de utilidad para la posteridad.

\section{Conclusiones}

Con el trabajo realizado se provee a las diferentes organizaciones de una metodología para la implementación de un sistema de calidad documentado de acuerdo con las normas ISO 9000:2000 y que brinde el soporte básico de su sistema de gestión de la calidad.

En el país se han observado acciones concretas encaminadas hacia los procesos de certificación de normativas internacionales de estandarización de parte de empresas de prestigio, logrando con esto desarrollar una ventaja competitiva palpable (Café Soluble S.A., Plastinic S.A., Nicaragua Sugar Estates Limited); para otros casos, el mayor beneficio, según lo expresa el encargado del proceso, ha sido en el área de desarrollo organizacional, como es el caso de Inversiones Papeleras S.A., que asegura haber reducido costos de operación y volúmenes de reproceso y no conformidades.

Sin embargo, la certificación ISO ha sido un tanto excluyente. Las empresas que han decidido involucrarse en el proceso son organizaciones que se derivan de una empresa multinacional y los lineamientos de su casa matriz lo indican siguiendo una filosofía organizacional encaminada hacia la conservación de la calidad. En otros casos, el departamento de calidad y la gerencia ejecutiva de la empresa están muy desarrolladas y poseen una plena conciencia de la necesidad de llegar a ser competitivos ante una transformación de la economía hacia 
la globalización.

Los esfuerzos de parte de la micro, pequeña y mediana empresa en obtener certificación de procesos basados en normativas internacionales no han sido sustanciales debido, en gran parte, al desconocimiento de la existencia de la herramienta $y$, en muchos casos, porque el proceso de implantación de la certificación ISO 9000 es demasiado costoso. Algunas empresas de tamaño medio han sido favorecidas por el proyecto BID-CADIN, que tiene como objetivo promover el desarrollo organizacional de la industria nacional, apoyando los procesos de acreditación a las diferentes empresas.

La principal cláusula de la normativa ISO 9000 es "Escribir lo que se hace", "Hacer lo que se dice", "Documentar lo que se ha hecho" y "Auditar para confirmar su cumplimiento". Cumplir este procedimiento asegurará el éxito en cada una de las etapas de implantación de la norma, si involucra y motiva a la totalidad de la fuerza laboral, iniciando por la alta dirección.

Se dice que los países llamados actualmente en vías de desarrollo son los que, por razones históricas, no participaron en la Revolución Industrial. Los efectos económicos de no participar en la llamada "Revolución Tecnológica" serán aún más graves que los sufridos por el país por no haber participado en la Revolución Industrial. Las normas ISO 9000 colocarían al país automáticamente en sintonía con la competitividad comercial y, por ende, con el desarrollo de las naciones.

\section{Referencias bibliográficas}

-BUREAU VERITA, (2000). Como obtener la certificación internacional de calidad ISO 9000 , Nicaragua

-BULLTEK LTD., (2003). Normativa ISO 9000 y Variantes, Sitio Oficial de Bulltek ltd., Disponible en http://www.bulltek.com, accesado el 23 mayo

-COY VERGARA, A. (2001). Sistema de gestión de la prevención de riesgos laborales: OHSAS 18001. Disponible en http://prevenycalidad.en.eresmas.com/ohsas.htm; accesado el 22 Mayo de 2003.

-EQA, (2003). ¿Que es EQA?, Sitio oficial de EQA en español Disponible en http:/www.eqa. es/, accesado el 23 de mayo

-ISOMAC CONSULTANTS,(2003). SA 8000-Sistema de Gestión de la Responsabilidad Social. Sitio oficial de Isomac consultants. Disponible en http://www.isomac.com.ar/ 8000.htm; accesado el 22 Mayo

-PÉREZ RODRÍGUEZ, Z., (2001). Metodología para la implementación de un sistema documental ISO 9000, Sitio oficial de Monografías.com, disponible en www.monografias. com, accesado el 22 Mayo del 2003

-PÓVEDA SALVATIERRA., J. A., (2002). “Mejorando la Calidad de Nuestra Empresa”, La Prensa, pág. 4B, Managua, Octubre 9 